\title{
Experimental gonococcal infection in male volunteers: cumulative experience with Neisseria gonorrhoeae strains FA1090 and MS11mkC
}

\author{
Marcia M. Hobbs ${ }^{1,2}{ }^{*}$, P. Frederick Sparling ${ }^{1,2}$, Myron S. Cohen ${ }^{1,2}$, William M. Shafer ${ }^{3,4}$, Carolyn D. Deal ${ }^{5}$ and \\ Ann E. Jerse ${ }^{6}$
}

${ }^{1}$ Departments of Medicine, University of North Carolina, Chapel Hill, NC, USA

${ }^{2}$ Department of Microbiology and Immunology, University of North Carolina, Chapel Hill, NC, USA

${ }^{3}$ Department of Microbiology and Immunology, Emory University School of Medicine, Atlanta, GA, USA

${ }^{4}$ Laboratories of Bacterial Pathogenesis, Veterans Affairs Medical Center (Atlanta), Decatur, GA, USA

${ }^{5}$ National Institute of Allergy and Infectious Diseases, National Institutes of Health, Bethesda, MD, USA

${ }^{6}$ Department of Microbiology and Immunology, Uniformed Services University of the Health Sciences, Bethesda, MD, USA

Edited by:

Cynthia N. Cornelissen, Virginia

Commonwealth University School of

Medicine, USA

Reviewed by:

Stanley Spinola, Indiana

University-Purdue University at

Indianapolis, USA

Catherine Ann Ison, Health Protection

Agency, UK

\section{*Correspondence:}

Marcia M. Hobbs, Department of

Medicine, University of North

Carolina, CB\# 7031, Chapel Hill, NC

27599, USA.

e-mail:mmhobbs@med.unc.edu
Experimental infection of male volunteers with Neisseria gonorrhoeae is safe and reproduces the clinical features of naturally acquired gonococcal urethritis. Human inoculation studies have helped define the natural history of experimental infection with two wellcharacterized strains of $N$. gonorrhoeae, FA1090 and MS11mkC. The human model has proved useful for testing the importance of putative gonococcal virulence factors for urethral infection in men. Studies with isogenic mutants have improved our understanding of the requirements for gonococcal LOS structures, pili, opacity proteins, IgA1 protease, and the ability of infecting organisms to obtain iron from human transferrin and lactoferrin during uncomplicated urethritis. The model also presents opportunities to examine innate host immune responses that may be exploited or improved in development and testing of gonococcal vaccines. Here we review results to date with human experimental gonorrhea.

Keywords: gonorrhea, pathogenesis, infection, urethritis

\section{INTRODUCTION}

In nature, gonococcal infection is strictly limited to the human host. Uncomplicated infection can be asymptomatic or manifest as urethritis in men and cervicitis in women. Complications resulting from tissue invasion include orchitis, epididymitis and pelvic inflammatory disease, and systemic dissemination can lead to arthritis, tenosynovitis, or dermatitis. Like other inflammatory mucosal infections, gonorrhea also facilitates HIV transmission and acquisition. Treatment options for gonococcal infection are dwindling as worldwide, Neisseria gonorrhoeae is becoming increasing resistant to currently available antibiotics (Lewis, 2010). Experimental human urethral infection offers the potential to better understand the contributions of microbial factors and host immune responses to infection that may eventually translate into the development of an effective gonococcal vaccine and provides an appropriate model for vaccine testing.

The use of live N. gonorrhoeae to cause experimental infection has a long and sometimes sordid history dating back to the late eighteenth century when British surgeon John Hunter repeatedly inoculated patients with "venereal matter" to test whether venereal disease was infectious (Hunter, 1835). Recent reports of experimental gonorrhea studies conducted during the 1940s in vulnerable populations who were often deceived and infected without informed consent highlight the ethical issues that surround clinical research with human subjects (Frieden and Collins, 2010); the issues are particularly sensitive for experimental human infections with sexually transmitted pathogens. All of the studies described herein were subject to rigorous safety and ethical review by appropriate Institutional Review Boards. Written informed consent was obtained from all subjects in accordance with the ethical principles set forth in the Declaration of Helsinki and the US Code of Federal Regulations for the Protection of Human Subjects. Several hundred subjects have participated in experimental infection studies without reported complications or adverse events.

\section{EXPERIMENTAL MODEL}

Experimental gonorrhea is restricted to male subjects; women cannot safely be included due to potential complications from ascendant gonococcal infection. Inoculation is accomplished by the instillation of $0.2-0.3 \mathrm{~mL}$ of a gonococcal suspension through a pediatric catheter inserted approximately $5 \mathrm{~cm}$ into the urethra. There are slight variations in the preparation and delivery of experimental inocula used in previously published work detailed by Cohen and Cannon (1999), Cohen et al. (1994), and Schmidt et al. (2001). Following inoculation, infection can be monitored by bacterial recovery from urogenital specimens including urine, urethral swabs and semen. Gonococcal urethritis is defined by the development of urethral discharge containing gram negative diplococci or recovery of gonococci from a urethral swab culture. Host responses can be monitored by enumeration of white blood cells in urine and urethral exudates and measurement of soluble immune mediators including antibodies and cytokines in urine 
and serum. Clinical disease is monitored by daily physical examination. Subjects receive prompt effective antibiotic treatment as soon as infection is clinically apparent, when requested because of intolerance for symptoms or at the end of the experimental trial, up to 7 days after inoculation, regardless of whether or not the subject was judged to be infected during the trial.

Two gonococcal strains, N. gonorrhoeae FA1090 and MS11mkC, have been used in the majority of experimental infection studies conducted over the past 20 years. FA1090 is a porin serotype PIB-3 strain originally isolated in the 1970s from the endocervix of a woman with probable disseminated gonococcal infection (Nachamkin et al., 1981) and has been used extensively in experimental infection studies conducted at the University of North Carolina at Chapel Hill (Cohen et al., 1994; Jerse et al., 1994; Seifert et al., 1994; Cornelissen et al., 1998; Cohen and Cannon, 1999; Johannsen et al., 1999). MS11 is a porin serotype PIB-9 strain originally isolated in 1970 from a patient with uncomplicated anterior urethritis (Edwards et al., 1984; Swanson et al., 1985). MS11mk is descended from MS11, and MS11mkC is a lipooligosaccharide (LOS) variant isolated from an experimentally infected male volunteer in the late 1980s (Schneider et al., 1991). MS11mkC was used in subsequent volunteer studies conducted at the Walter Reed Army Institute of Research (Ramsey et al., 1995; Schneider et al., 1995, 1996; Schmidt et al., 2000, 2001).

\section{N. GONORRHOEAE MS11mkC IS MORE INFECTIOUS THAN FA1090 IN THE MALE URETHRA}

Too few subjects can be included in individual experimental trials to reliably evaluate the infectivity of a given inoculum. However, the cumulative experience to date includes sufficient numbers of subjects and a wide enough range of doses to estimate the relative infectivities of the two strains. We analyzed available data from 55 men inoculated with wild-type MS11mkC at doses from $2.5 \times 10^{2}$ to $5.7 \times 10^{4}$ colony-forming units (cfu; Schneider et al., 1995; Schmidt et al., 2001 and unpublished data from J. G. Cannon) and 43 men inoculated with wild-type FA1090 at doses from $1.0 \times 10^{4}$ to $3.5 \times 10^{6} \mathrm{cfu}$ (Cohen et al., 1994 and unpublished data from J. G. Cannon and M. M. Hobbs). We conducted multiple logistic regression analyses using SigmaStat version 3.5 for Windows (Systat Software, Inc., Point Richmond, CA, USA) to predict the probability of infection over a range of doses for each strain (Figure 1). The quality of the fit for each dose response curve was tested using the Hosmer-Lemeshow statistic (Hosmer and Lemeshow, 2000). A non-significant $P$ value $(>0.05)$ indicates a good fit between the logistic equation and the raw data; $P=0.369$ for MS11mkC, and $P=0.878$ for FA1090. The estimated dose resulting in infection of $50 \%$ of inoculated subjects $\left(\mathrm{ID}_{50}\right)$ is $1.8 \times 10^{3} \mathrm{cfu}$ for MS11 mkC and $1.0 \times 10^{5} \mathrm{cfu}$ for FA1090. Similar differences in infectivity for the two strains have been observed in the murine model of female genital tract infection (Jerse, 1999).

The reasons for the apparent nearly $2 \log _{10}$ difference in $\mathrm{ID}_{50}$ between FA1090 and MS11 mkC for urethral infection are not clear. Great care has been taken to describe and control (as much as possible) the expression of recognized phase-variable determinants in experimental inocula. Inoculum variants of both strains used in the experimental infections included in the dose response analyses

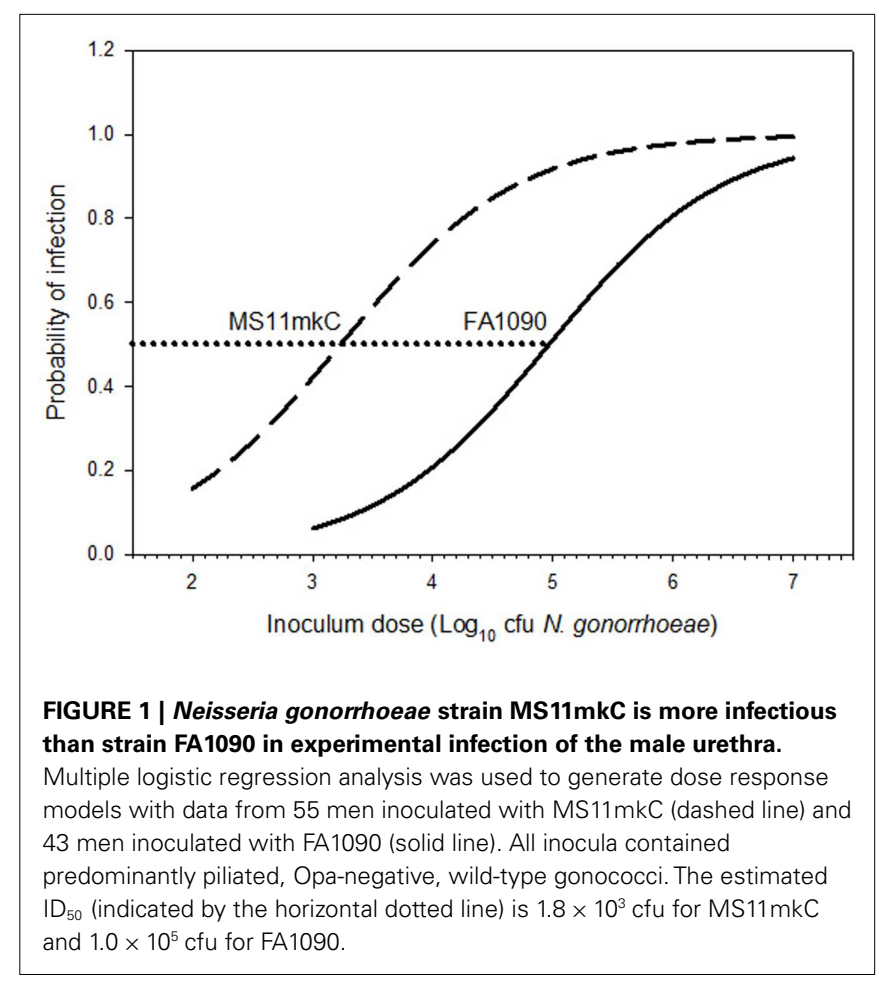

contained predominantly piliated $(\mathrm{P}+)$, Opacity protein-negative $(\mathrm{Opa}-)$ gonococci expressing the lacto- $N$-neotetraose LOS epitope recognized by monoclonal antibody (Mab) 3F11, which is associated with the development of urethral discharge in naturally acquired and experimental gonococcal infection in men (Schneider et al., 1991). Strains FA1090 and MS11 have been extensively characterized in numerous laboratories, and there are many recognized differences. Though not an exhaustive list, Table 1 summarizes some of the features that differ between FA1090 and MS11mkC, potentially influencing infectivity in the experimental model of urethral infection.

The relatively recent human passage of MS11mkC compared to FA1090 may also have resulted in unrecognized differences between the two strains. Though inocula prepared from both strains have been described as piliated, it is possible that MS11 mkC is hyperpiliated compared to FA1090.

\section{NATURAL HISTORY OF EXPERIMENTAL INFECTION WITH WILD-TYPE GONOCOCCI}

Despite the apparent difference in infectivity, the course of experimental infection and development of clinical signs and symptoms caused by wild-type piliated, Opa-negative inocula of N. gonorrhoeae strains FA1090, and MS11mkC are very similar. The clinical and microbiological courses of infection in individual infected subjects have been described previously (Schneider et al., 1991, 1995, 1996; Cohen et al., 1994; Jerse et al., 1994; Cohen and Cannon, 1999; Schmidt et al., 2001) and are summarized here. Gonococci are recovered at low levels in urine from most subjects $2 \mathrm{~h}$ after inoculation. A so-called eclipse period of variable length ensues during which few or no viable gonococci are recovered from urine. Then, infected individuals begin to 
Table 1 | Recognized differences between N. gonorrhoeae strains FA1090 and MS11mkC.

\begin{tabular}{|c|c|c|c|}
\hline Characteristic & FA1090 & MS11mkC & References \\
\hline Serum susceptibility & Resistant & $\begin{array}{l}\text { Sensitive (characterized as } \\
\text { Intermediate by some) }\end{array}$ & $\begin{array}{l}\text { Cohen et al. (1994), } \\
\text { Ram et al. (2001) }\end{array}$ \\
\hline Gonococcal genetic island & Absent & Present & Dillard and Seifert, (2001) \\
\hline $\begin{array}{l}\text { Lactoferrin utilization (expression of } \\
\text { lactoferrin-binding proteins } B \text { and } A \text { ) }\end{array}$ & Lf- (LbpB-A-) & $\mathrm{Lf}+(\mathrm{LbpB}-\mathrm{A}+)$ & Anderson et al. (2003) \\
\hline Mtr efflux pump expression & $\begin{array}{l}\text { Not inducible due to } 11 \text { bp deletion } \\
\text { in the coding region of activator } \\
m \operatorname{tr} A \text {; wt } m \operatorname{tr} C D E \text { promoter }\end{array}$ & $\begin{array}{l}\text { Inducible with wt mtrA; } \\
\text { higher expression of pump } \\
\text { due to novel } m \operatorname{tr} C D E \\
\text { promoter and missense } \\
\text { mutation in repressor } m t r R\end{array}$ & $\begin{array}{l}\text { Rouquette et al. (1999), Warner } \\
\text { et al. (2008), and W. M. Shafer } \\
\text { (unpublished data) }\end{array}$ \\
\hline
\end{tabular}

Lf, lactoferrin; Lbp, lactoferrin-binding protein; Mtr, multiple transferable resistance; bp, base pair; wt, wild-type.

shed increasing numbers of organisms in the urine, though the number of gonococci recovered from infected subjects does not correlate with the severity of infection. Bacteriuria persists until signs and symptoms of infection including dysuria and urethritis develop. Most men experimentally infected with wild-type gonococci develop gonococcal disease characterized by a urethral discharge and dysuria, consistent with the clinical presentation of naturally acquired gonorrhea. The incubation period between inoculation and development of signs or symptoms of infection is variable, ranging from 1 to 6 days for both FA1090 and MS11mkC and is independent of the number of gonococci in the inoculum. Though rare, asymptomatic experimental infection with wild-type FA1090 variants occurs and is characterized by recovery of gonococci from urine in the absence of signs or symptoms during the 5- to 6-day trial period (J. G. Cannon and A. E. Jerse, unpublished data); asymptomatic infection with MS11mkC has not been reported.

Cellular and soluble mediators of the host response to experimental infection follow a similar pattern. Pyuria, as determined by the presence of white blood cells in urine sediment (Cohen et al., 1994; Ramsey et al., 1995), and elevated urinary interleukin (IL)-6 and IL-8 (Ramsey et al., 1995) are frequently observed within the first $24 \mathrm{~h}$ after inoculation. Whether the early appearance of these inflammatory mediators is the result of mild irritation caused by the insertion of the catheter during inoculation or a direct response to gonococci is not clear. In subjects who remain uninfected, pyuria resolves (Cohen et al., 1994; Ramsey et al., 1995), and cytokines quickly return to pre-challenge levels (Ramsey et al., 1995). Pyuria persists in infected subjects, and the numbers of white blood cells in urine generally increase until men develop symptoms and require treatment (Cohen et al., 1994; Ramsey et al., 1995). Elevated levels of inflammatory cytokines IL-6, IL- 8, TNF $\alpha$ and IL- $1 \beta$ are consistently detected in urine in response to experimental infection with MS11 mkC or MS11mkA, (an LOS variant lacking the 3F11 epitope; Schneider et al., 1991; Ramsey et al., 1995); similar cytokine studies have not been conducted with strain FA1090. The cytokine responses detected in urine during experimental gonorrhea are comparable to those seen during bacterial urinary tract infections (Sheu et al., 2006, 2007). During experimental gonococcal urethritis, inflammatory host response indicators generally peak at the onset of symptoms and return to pre-inoculation levels within 2 days after treatment (Ramsey et al., 1995). Elevated inflammatory cytokines in peripheral circulation are also detected during experimental infection, returning to baseline after treatment. Cytokines in urine are uniformly detectable in infected subjects with elevated plasma cytokines. However, elevated urinary cytokine levels in the absence of concomitant increases in plasma in some infected subjects suggest local involvement in inflammatory responses to experimental urethral infection (Ramsey et al., 1995).

Because experimentally infected subjects are treated at the onset of signs or symptoms of infection, assessment of immune responses is limited to those that occur during the early stages of infection such as the innate immune responses described above. Acquired immune responses including production of specific antibodies are generally thought to require longer exposure than occurs during experimental infection. However, Schneider and colleagues demonstrated increases in serum IgG antibodies recognizing gonococcal LOS in 8 of 14 subjects (57\%) experimentally infected for 3-7 days with an ID $_{90}$ of MS11mkC (Schmidt et al., 2001). Interestingly, increased anti-LOS antibody titers were significantly associated with resistance to homologous reinfection, though equivalent proportions of previously infected and naïve subjects were infected with an $\mathrm{ID}_{50}$ challenge (Schmidt et al., 2001). Men who experienced a fourfold or greater increase in antiLOS IgG titer during initial infection were more likely to resist reinfection than men with lower and static LOS antibody titers during initial infection. The mechanisms by which anti-LOS antibodies in serum could protect from urethral gonococcal infection are not clear. Increases in these circulating antibodies may be a surrogate for protective mucosal immune responses, or transudation to the urethral mucosa could result from early inflammation in experimental infection. Apicella and colleagues have shown that gonococcal entry into primary human urethral epithelial cells is dependent on LOS interactions with the asialoglycoprotein receptor (Harvey et al., 2001), and van Kooyk and colleagues demonstrated that gonococcal LOS influences dendritic cell function and subsequent immune responses that promote bacterial survival (van Vliet et al., 2009). Mucosal anti-LOS antibodies could interfere with these processes at very early stages of infection and 
potentially result in host immune responses that favor bacterial clearance.

\section{EXPRESSION AND ANTIGENIC VARIATION OF GONOCOCCAL SURFACE STRUCTURES IN VIVO}

The remarkable variation in surface structures expressed by different $N$. gonorrhoeae strains and the extraordinary plasticity of the bacterial surface that an individual strain can present to its infected host are hallmarks of the gonococcus that likely contribute to high rates of recurrent gonococcal infection. The frequent modulation of bacterial surface determinants results from a combination of phase variation (the switch between on and off states of gene expression) and antigenic variation (the expression of different antigenic versions of a structure) that result in differential expression of outer membrane components including pili, Opa proteins, and LOS (reviewed in Edwards and Apicella, 2004). The genetic mechanisms that result in phase and antigenic variation have been extensively characterized (reviewed in Simms and Jerse, 2005), and in vitro models of gonococcal interactions with host cells indicate that different antigenic versions of pilin (the major pilus subunit), Opa proteins, and LOS recognize different host cell receptors, consistent with differential tissue tropism and pathogenesis of naturally occurring gonococcal variants (Virji, 2009). Phenotypic variation of these surface determinants occurs during experimental infection in the male urethra.

\section{LOS VARIATION AND MODIFICATION DURING EXPERIMENTAL URETHRAL INFECTION}

Production of multiple LOS structures occurs within a gonococcal strain as well as within individual colonies of a given strain. Alterations in LOS structures result from phase-variable production of enzymes involved in extending carbohydrate structures from the $\alpha$ - and $\beta$-chains of the core oligosaccharide. Variation in $\alpha$-chain residues is due to changes in polynucleotide repeat elements in the $\lg t A$, $\lg t C$, and $\lg t D$ genes, encoding glycosyl transferases, organized in an operon that also includes $\lg t B$ and $\lg t E$, whereas $\beta$-chain variation is due to alterations in a polynucleotide repeat in $\lg t G$ (Banerjee et al., 1998). The different LOS structures resulting from "phase-on" or "phase-off" expression of these genes, as well as variable modifications of the oligosaccharide core (e.g., attachment of phosphoethanolamine [PEA] to heptose I and II), can impact the biologic properties of gonococci, altering bacterial susceptibility to mediators of innate and acquired immune host defense and attachment to host cells. A critical question is whether different structures provide an advantage for gonococci during infection and at different stages (e.g., before or after development of inflammation). The importance of the lacto- $N$-neotetraose paraglobosyl epitope of gonococcal LOS was recognized early in the history of experimental infection with strain MS11mk. Following inoculation of male volunteers with MS11mkA, which only produced a 3.6-kD lactosyl LOS and lacked higher molecular weight gangliosyl and paraglobosyl structures, gonococci isolated at the onset of dysuria expressed gangliosyl LOS moieties, and bacteria isolated at the onset of urethral discharge (including the MS11mkC variant) expressed paraglobosyl structures (Schneider et al., 1991; John et al., 1999). Gonococci isolated from symptomatic men with naturally acquired gonorrhea also widely express gangliosyl or paraglobosyl LOS, but rarely express the lactosyl moiety (Schneider et al., 1991). In the MS11 background, expression of paraglobosyl LOS increased the apparent infectivity of the $\mathrm{mkC}$ variant compared to the mkA variant $(5 / 5 \mathrm{vs.} 2 / 5$ inoculated volunteers infected with $4 \times 10^{4} \mathrm{cfu}$, Schneider et al., 1995), suggesting that this structure is important in the pathogenesis of gonococcal urethritis. However, N. gonorrhoeae strain FA1090, which is less infectious than MS11mkC in the human model, also expresses a paraglogosyl LOS. Thus, the relative infectivity of the two different strains cannot be attributed to expression of this structure.

Taken together, the observations described above suggest that gonococci producing gangliosyl or paraglobosyl LOS species are at a selective advantage during infection of the male urethra. To directly test this idea, Shafer and colleagues are constructing a variant of N. gonorrhoeae FA1090 that should not express gangliosyl or paraglobosyl LOS due to an internal deletion that removes most of $\lg t A$ and $\lg t C$ and all of $\lg t B$. This mutant should only express the 3.6-kD lactosyl LOS structure. Competitive experimental infections with inocula containing this non-variable LOS mutant and wild-type strain FA1090, which can freely phase vary its LOS structures, could determine whether phase-variable production of LOS structures is important during infection. If the ability to alter LOS structures during urethral infection is advantageous, the "lockedin" mutant is predicted to have a fitness defect or disadvantage in vivo.

The lipid A core of gonococcal LOS has endotoxic activity, and recent evidence indicates that PEA modification at the $4^{\prime}$ position is an important determinant in the capacity of strains to resist complement-mediated killing by normal human serum (Lewis et al., 2009; Balthazar et al., 2011). The presence of PEA can also enhance resistance to cationic antimicrobial peptides. Interestingly, PEA modification at heptose II does not impart such resistance. Shafer and co-workers have constructed an lptA deletion mutant of N. gonorrhoeae strain FA1090 (W. M. Shafer, unpublished data). Mixed experimental infections with this mutant, which cannot modify lipid A with PEA, and wild-type FA1090 will test whether PEA-containing lipid A provides a fitness advantage to gonococci during urethral infection.

The terminal paraglobosyl oligosaccharide recognized by Mab $3 \mathrm{~F} 11$ and expressed by MS11mkC and FA1090 variants used in experimental infection studies to date can be modified by gonococcal sialyltransferase, resulting in the addition of host-derived sialic acid to the bacterial surface, mimicking human cell surface antigens (Mandrell et al., 1988, 1990; Apicella et al., 1990; Parsons et al., 1994). Sialylation of gonococcal LOS in vitro increases serum resistance of intrinsically serum sensitive strains (Griffiss et al., 1991), promotes gonococcal resistance to killing by human neutrophils (Kim et al., 1992; Rest and Frangipane, 1992; Wetzler et al., 1992; Gill et al., 1996), and protects N. gonorrhoeae from the bactericidal activity of porin and LOS-specific antibodies (Parsons et al., 1989; Elkins et al., 1992; Wetzler et al., 1992; Gill et al., 1996). Furthermore, immunoelectron microscopic analysis of gonococci in urethral secretions from men with naturally acquired gonorrhea indicates that sialylation occurs during infection (Apicella et al., 1990). However, sialylation of MS11 mkC prior to urethral inoculation appears to reduce infectivity of this strain and lengthen 
the incubation period for development of symptomatic urethritis (Schneider et al., 1996). Sialylation also reduces gonococcal invasion of primary urethral epithelial cells in vitro (Harvey et al., 2001). These observations suggest that gonococcal sialylation in the male urethra may occur after the initial stages of infection. The presence or absence of sialylation of LOS may also be important for bacterial survival during different phases of infection or in different compartments during infection. For example, nonsialylated gonococci may more easily enter urethral epithelial cells, whereas sialylation maybe provide protection from the bactericidal effects of transudated serum that could affect extracellular bacteria on the urethral mucosa. N. gonorrhoeae likely alternates between intracellular and extracellular compartments during infection, and variable LOS sialylation may facilitate transitions between these compartments.

Sialyltransferase (lst) mutants of $N$. gonorrhoeae MS11mkC and FA1090 have been tested in the experimental human model, and $l s t$-deficient gonococci from both backgrounds are able to cause infection in the male urethra (J. G. Cannon, unpublished data). After inoculation with an lst null mutant in the serum resistant FA1090 background at an approximate $\mathrm{ID}_{80}$ for wild-type FA1090, three out of four subjects became infected. FA1090lst gonococci were recovered from the urine of all three infected subjects; two developed urethral discharge during the 5-days after inoculation, and the third remained asymptomatic during the experimental trial. In the relatively serum sensitive MS11mkC background at an approximate $\mathrm{ID}_{80}$ for the wild-type strain, four out of eight subjects became infected after inoculation with an lst null mutant. MS11mkClst gonococci were recovered from the urine of all four infected subjects; two developed urethral discharge during the 5-days after inoculation, and the other two infected subjects remained asymptomatic during the experimental trial.

Because asymptomatic experimental infection with wild-type MS11mkC is rare, the lack of sialyltransferase activity may have altered the pathogenesis of this strain in experimental urethral infection. However, experimental human infection trials with small numbers of subjects receiving individual inocula containing only mutant or wild-type gonococci often does not provide sufficient power to demonstrate reduced infectivity or pathogenesis with statistical confidence. In experimental infection of female mice inoculated with wild-type MS11 or an isogenic lst mutant,
Wu and Jerse saw no significant difference in infectivity, consistent with the results of experimental human infection. Using more sensitive competitive infections in mice inoculated with mixtures of wild-type gonococci and similar numbers of the lst mutant, bacteria lacking $l s t$ were significantly attenuated in the capacity to colonize the lower female murine genital tract (Wu and Jerse, 2006). Competitive infections with mixed inocula in experimental human infection may reveal an advantage for expression of sialyltransferase in MS11 and/or FA1090 in the male urethra that was not apparent in previous infections with pure inocula.

The effects of differences in LOS expression on experimental $N$. gonorrhoeae infection of male volunteers are summarized in Table 2.

\section{OPACITY (OPA) PROTEIN EXPRESSION DURING EXPERIMENTAL URETHRAL INFECTION}

Individual $N$. gonorrhoeae strains possess 11 or 12 opa genes, each of which is subject to reversible, high frequency changes in expression state. As a result, individual gonococci can express one or multiple different Opa proteins, or none at all. Sequence diversity in regions of opa genes encoding antigenic determinants exposed on the bacterial surface confers specificities for different receptors on human cells. Most Opa variants bind to human carcinoembryonic antigen cell adhesion molecule (CEACAM) family receptors, however a small number of Opa proteins bind heparin sulfate proteoglycans (HSPGs). Opa-mediated interactions between gonococci and host cells can activate neutrophils or suppress activation and proliferation of CD4 $+\mathrm{T}$ lymphocytes, depending on which receptors are engaged (reviewed in Sadarangani et al., 2011). Opa protein repertoires of different $N$. gonorrhoeae strains differ in primary amino acid sequences, and the nomenclature in the literature describing individual Opa proteins in different strains is ambiguous. The opa genes of strains FA1090 and MS11 show little homology in the variable regions encoding antigenic portions of the proteins (Connell et al., 1990; Bhat et al., 1991), and proteins referred to as OpaA, OpaB, etc., are not the same in the two strains.

Early observations by James and Swanson (1978) documented that gonococci isolated from men with urethritis predominantly formed the characteristically opaque colonies associated with Opa protein expression. There is also strong selection for expression of Opa proteins in the male urethra following inoculation with wild-type variants of FA1090 or MS11 mkC that are predominantly

Table 2 | Effects of lipoologosaccharide (LOS) features on experimental gonorrhea.

\begin{tabular}{|c|c|c|c|}
\hline Phenotype tested & Strain background & Experimental outcome & References \\
\hline $\begin{array}{l}\text { Natural phase variation } \\
\text { resulting in changes in LOS } \\
\text { structures }\end{array}$ & MS11mkC & $\begin{array}{l}\text { Expression of paraglobosyl structures } \\
\text { favored during infection }\end{array}$ & Schneider et al. (1995) \\
\hline \multirow[t]{2}{*}{$\begin{array}{l}\text { Ability to sialylate paraglobosyl } \\
\text { LOS structures }\end{array}$} & FA1090 (serum resistant) & $\begin{array}{l}\text { Sialyltransferase (/st) mutant as } \\
\text { infectious as wild-type with pure inocula }\end{array}$ & J. G. Cannon (unpublished data) \\
\hline & $\begin{array}{l}\text { MS11mkC (serum } \\
\text { sensitive) }\end{array}$ & $\begin{array}{l}\text { Trend toward lower infectivity for Ist } \\
\text { mutant compared to wild-type with pure } \\
\text { inocula }\end{array}$ & J. G. Cannon, (unpublished data) \\
\hline
\end{tabular}

LOS, lipooligosaccharide; Ist, gene encoding gonococcal sialyltransferase. 
Opa-negative (Jerse et al., 1994; Schmidt et al., 2000). Schneider and colleagues showed that transition from Opa-negative to Opapositive MS11mkC gonococci shed by experimentally infected men is associated with the onset of symptoms (Schneider et al., 1995, 1996; Schmidt et al., 2000). Using strain FA1090, Jerse et al. (1994) showed that not only are Opa+ variants uniformly isolated from urine and urethral swab cultures from experimentally infected men, the proportion of isolates expressing multiple Opa proteins increases over time. No single Opa protein in either strain's repertoire is selectively expressed during experimental infection; different Opas predominated among colonies isolated from individual subjects. However, there are Opa proteins in each strain's repertoire that appear to be under-represented among isolates from experimentally infected subjects (Jerse et al., 1994; Schmidt et al., 2000).

Following inoculation with predominantly Opa-negative $N$. gonorrhoeae FA1090, variants expressing this strain's OpaA alone were not isolated from any of the subjects, and only a small number of reisolates expressed OpaA in conjunction with one or more other Opa proteins (Jerse et al., 1994). Opa-expressing FA1090 have also been used to initiate experimental urethral infections. Inoculation with $10^{4} \mathrm{cfu}$ of FA1090 expressing predominantly OpaA (along with the 3F11 LOS epitope and 98.8\% identical pilin amino acid sequence compared to the Opa-negative inoculum variant) resulted in infection in four out of five subjects, and three of the four infected subjects developed acute urethritis within 1-3 days after inoculation. The fourth subject shed gonococci in his urine, but remained asymptomatic during the 4-day trial. Gonococci isolated at the onset of signs of acute urethritis resulting from the predominantly OpaA inoculum continued to express OpaA, but in conjunction with one or more other Opa proteins. Gonococci expressing multiple Opa proteins represented a markedly higher proportion of isolates recovered from subjects inoculated with FA1090 OpaA (mean 96.7\%, range $92-100 \% ; n=3$ subjects) than was observed with predominantly Opa-negative inocula (mean $47.8 \%$; range $18-100 \%$; $n=9$ subjects) or with an FA1090 variant expressing predominantly OpaF (mean 28.3\%; range 7-75\%; $n=3$ subjects; J. G. Cannon and A. E. Jerse, unpublished data). In contrast to FA1090 OpaA, the OpaF protein was highly represented among reisolates from subjects inoculated with Opa-negative FA1090 (Jerse et al., 1994). Thus, gonococci expressing OpaA alone appeared to be at a disadvantage during experimental urethral infection.

Rest and colleagues demonstrated that, in contrast to other Opa variants of FA1090, OpaA variants are resistant to neutrophil killing in vitro (Fischer and Rest, 1988; Elkins and Rest, 1990). The relevance of in vitro Opa-mediated stimulation of neutrophils to urethral pathogenesis is not clear. One might predict that resistance to neutrophil killing would provide an advantage for OpaA expression during infection. However, the rarity of OpaA expression following inoculation with Opa-negative gonococci, and the additional expression of other Opa proteins following inoculation with OpaA expressers confound this hypothesis, as presumably multiple expressers would be killed by neutrophils. These results suggest that FA1090 OpaA may not mediate key function(s) required for gonococcal survival in the male urethra.
The rapid and uniform shift from Opa-negative to Opa protein expression by gonococci in the male urethra exhibited by both wild-type strains FA1090 and MS11mkC suggested that the capacity to express opacity proteins may be required during urethral infection, and an Opa-deficient FA1090 mutant was predicted to be attenuated in the human experimental model. FA1090opaA$K$ is a genetically defined mutant in which all 11 opa genes of this strain have been inactivated (Cole et al., 2010). Inoculation with FA1090opaA-K at doses ranging from 5.5 to $6.4 \log _{10} \mathrm{cfu}$ resulted in infection in four out of eight subjects (J. G. Cannon, unpublished data). FA1090opaA-K gonococci were recovered from the urine of all four infected subjects; three of the four developed urethral discharge within 4 days after inoculation, the fourth remained asymptomatic during the 5-day experimental trial. Thus, Opa protein expression is not required for gonococcal infection in the male urethra.

Opa protein expression does confer an advantage in the late stages of female murine genital tract using FA1090opaA-K and a complemented strain that constitutively expresses OpaB, with statistically significant differences in colonization load observed on days 9-14 of infection (Cole et al., 2010). Whether a similar advantage for Opa expression could be demonstrated in the human male urethra is not clear. Experimental infection in men is limited to earlier stages of infection than can be studied using the murine model; an advantage for Opa protein expression that did not manifest until later stages of urethral infection could not be observed in the human model. The murine and human genital tracts differ in expression of receptors for Opa proteins, and the selective pressures influencing Opa protein expression in the male and female human genital tracts likely differ. Thus, the apparent advantage conferred by Opa expression in the female murine genital tract may not apply to urethral infection in men.

\section{PILIN EXPRESSION AND VARIATION IN VIVO}

Gonococcal type 4 pili are important for attachment to host cells, and phase and antigenic variation of PilE, the major pilin protein, are thought to provide the gonococcus with mechanisms of immune evasion and tissue tropism (reviewed in Virji, 2009). Gonococci recovered from men and women with symptomatic, naturally acquired gonorrhea are uniformly piliated (Kellogg et al., 1963, 1968). Early experimental infections of male volunteers (with N. gonorrhoeae strain F62) showed that piliated gonococci were infectious and produced purulent urethral exudates, and virulence was retained after extensive selective passage in vitro (Kellogg et al., 1963). In contrast, though non-piliated inocula were infectious, infected men developed a watery urethral discharge or none at all (Kellogg et al., 1968). Furthermore, after extensive passage in vitro, non-piliated F62 gonococci were noninfectious (Kellogg et al., 1963). Regardless of whether piliated or non-piliated inocula were used, gonococci recovered from infected subjects were uniformly piliated (Kellogg et al., 1963, 1968).

Rapid and extensive antigenic variation of PilE has been shown to occur during experimental urethral infection with N. gonorrhoeae FA1090 (Seifert et al., 1994; Wright et al., 1994; Hamrick et al., 2001) and MS11mk (Swanson et al., 1987). These 
observations suggested that type 4 pilus expression may be required for urethral gonococcal infection, and a non-reverting, non-piliated FA1090 mutant was predicted to be non-infectious in the human experimental model. The promoter and $5^{\prime}$ end of the single pilin expression locus was deleted in FA1090pilE (Cannon et al., 1996). Inoculation with approximately $10^{6} \mathrm{cfu}\left(\sim \mathrm{ID}_{80}\right.$ for piliated wild-type FA1090) of Opa-negative FA1090pilE expressing the paraglobosyl LOS epitope recognized by Mab 3F11 resulted in infection in six out of eight subjects (J. G. Cannon, unpublished data). Non-piliated gonococci were cultured from urine from all infected subjects. Three of the six subjects infected with the pilE mutant developed a watery urethral discharge, and the remaining subjects remained asymptomatic throughout the 5-day experimental trial, consistent with Kellogg et al. $(1963,1968)$ earlier observations with phase-variable, non-piliated inocula that switched to pilus expression during infection. These results suggest that host responses to piliated and non-piliated gonococci may differ during early stages of urethral infection. The fact that pilus expression is not required for infection in men suggests there may be a role for alternative adhesins in urethral colonization.

Although FA1090pile does not elaborate a pilus fiber, this mutant does express the pilus-associated PilC protein, which can be present both in pilus fibers and in the gonococcal outer membrane (Rudel et al., 1995; Rahman et al., 1997). PilC is important for adherence of piliated gonococci to human epithelial cells (Rudel et al., 1992; Nassif et al., 1994; Kirchner and Meyer, 2005), and in the absence of PilE, PilC has also been shown to increase adherence of FA1090 to epithelial cells in vitro, though the effect of PilC expression on non-piliated gonococci is modest compared to adherence of piliated bacteria (C. E. Thomas, unpublished data). Mixed experimental infections with FA1090pilE expressing PilC and a mutant that expresses neither PilE nor PilC may help elucidate the roles of these two proteins in gonococcal pathogenesis in the male urethra.

The effects of opacity protein and pilin expression on experimental N. gonorrhoeae infection of male volunteers are summarized in Table 3.

\section{IgA PROTEASE IS NOT REQUIRED DURING EXPERIMENTAL GONOCOCCAL URETHRITIS}

IgAl protease production is associated with mucosal pathogens including $N$. gonorrhoeae, N. meningitidis, and Haemophilus influenzae (Kilian et al., 1996). In addition to its ability to cleave IgA1 at mucosal surfaces, gonococcal IgA1 protease also cleaves the lysosome-associated membrane protein 1 (Lamp1) promoting intracellular bacterial survival in epithelial cells in vitro (Lin et al., 1997; Ayala et al., 2002). These observations suggested that the enzyme may contribute to gonococcal pathogenesis. Cannon and colleagues showed that a $N$. gonorrhoeae FA1090 iga mutant (Opa-, P+, and expressing the 3F11 LOS epitope) that lacks IgAl protease activity is fully capable of causing urethritis in male volunteers (Johannsen et al., 1999).

IgA1 protease could be more important in later stages of infection than can be assessed in the human model of uncomplicated urethritis in naïve volunteers. Protease function could contribute to later-stage invasive complications, or may play a role in reinfection of previously exposed individuals by inactivating pre-existing mucosal antibodies (Johannsen et al., 1999). The role of IgA1 protease in gonococcal infection in women has not been tested.

\section{IMPORTANCE OF TRANSFERRIN AND LACTOFERRIN RECEPTORS IN GONOCOCCAL PATHOGENESIS}

Like all N. gonorrhoeae strains, FA1090, and MS11mkC can use iron from human transferrin (Tf) for growth. One of the notable differences between these two strains (Table 1) is their differential ability to obtain iron from human lactoferrin (Lf). MS11mkC expresses lactoferrin-binding protein A (LbpA) and is phenotypically $\mathrm{Tf}+\mathrm{Lf}+$, whereas FA1090 has a naturally occurring deletion that eliminates expression of LbpA and is unable to grow on lactoferrin as a sole source of iron (Anderson et al., 2003). Thus, wild-type FA1090, like approximately half of clinical isolates of $N$. gonorrhoeae that have been examined (Mickelsen et al., 1982; Fox et al., 1998; Anderson et al., 2001), is phenotypically Tf+Lf-. Cornelissen et al. (1998) showed that a transferrin-receptor mutant of FA1090, which cannot obtain iron from either Tf or Lf, is incapable of causing urethritis in the experimental human gonorrhea model.

Table 3 | Effects of opacity protein (Opa) and pilin (PilE) expression on experimental gonorrhea.

\begin{tabular}{|c|c|c|c|}
\hline Phenotype tested & Strain background & Experimental outcome & References \\
\hline $\begin{array}{l}\text { Natural phase variation } \\
\text { resulting in changes in Opa }\end{array}$ & MS11mkC and FA1090 & $\begin{array}{l}\text { Opa expression favored during infection } \\
\text { after inoculation with Opa-negative }\end{array}$ & $\begin{array}{l}\text { Jerse et al. (1994), Schmidt et al. } \\
\text { (2000) }\end{array}$ \\
\hline expression & & variant & \\
\hline $\begin{array}{l}\text { Requirement for Opa } \\
\text { expression }\end{array}$ & FA1090 & $\begin{array}{l}\text { Mutant unable to express Opa proteins } \\
\text { as infectious as wild-type }\end{array}$ & J. G. Cannon (unpublished data) \\
\hline $\begin{array}{l}\text { Natural antigenic variation in } \\
\text { PilE protein }\end{array}$ & MS11mkC and FA1090 & $\begin{array}{l}\text { Extensive variation observed during } \\
\text { infection with piliated variant }\end{array}$ & $\begin{array}{l}\text { Seifert et al. (1994), Wright et al. } \\
\text { (1994), Hamrick et al. (2001), } \\
\text { Swanson et al. (1987) }\end{array}$ \\
\hline Requirement for PilE & FA1090 & Non-piliated mutant unable to express & J. G. Cannon (unpublished data) \\
\hline expression & & PilE as infectious as wild-type & \\
\hline
\end{tabular}

Opa, opacity protein; PilE, pilin protein 
Sparling and colleagues showed that a N. gonorrhoeae FA1090 engineered mutant capable of using Lf but not Tf, a combination not seen in nature, causes experimental urethral infection in male volunteers that is similar to infections caused by wild-type FA1090 (Anderson et al., 2003). Thus iron from either Tf or Lf enables gonococcal growth and pathogenesis in the male urethra.

To determine whether the ability to use human Lf provides an advantage to gonococci that also can use human Tf, Sparling and colleagues conducted competitive infections with wild-type FA1090 (Tf+Lf- $)$ and a Tf+Lf+ mutant. Inoculation with an approximate $\mathrm{ID}_{50}$ for wild-type FA1090 containing a mixture of equivalent numbers of both strains resulted in infection in nearly half of subjects. In each of five infected subjects, the Tf $+\mathrm{Lf}+$ strain exhibited a significant competitive advantage over wildtype FA1090; urine and urethral swab cultures from all subjects contained 100\% mutant gonococci at the end of the experimental trial (Anderson et al., 2003). Thus, the experimental model using a competitive infection study design has the potential to demonstrate differences in fitness between gonococcal strains with statistical confidence, even when the overall infectivity of the two strains is not demonstrably different.

The requirements for IgA protease and iron acquisition from human iron-binding proteins during experimental $N$. gonorrhoeae infection of male volunteers are summarized in Table 4.

\section{POPULATION DYNAMICS IN EXPERIMENTAL INFECTION}

Tracking the variation of opacity and pilin proteins during experimental infection with wild-type $N$. gonorrhoeae reveals that the phenotype of infecting bacteria changes multiple times in the interval between inoculation and the development of urethral discharge (Jerse et al., 1994; Seifert et al., 1994; Wright et al., 1994; Schmidt et al., 2000; Hamrick et al., 2001). As shown schematically in Figure 2, inoculum variants disappear rapidly and are replaced by a series of new variants expressing different combinations of surface antigens. The gonococcal population becomes more complex as infection proceeds, consisting of a mixture of multiple variants at the time a urethral discharge is present. These changes are consistent with sequential outgrowth and disappearance of clonal populations expressing combinations of Opa and

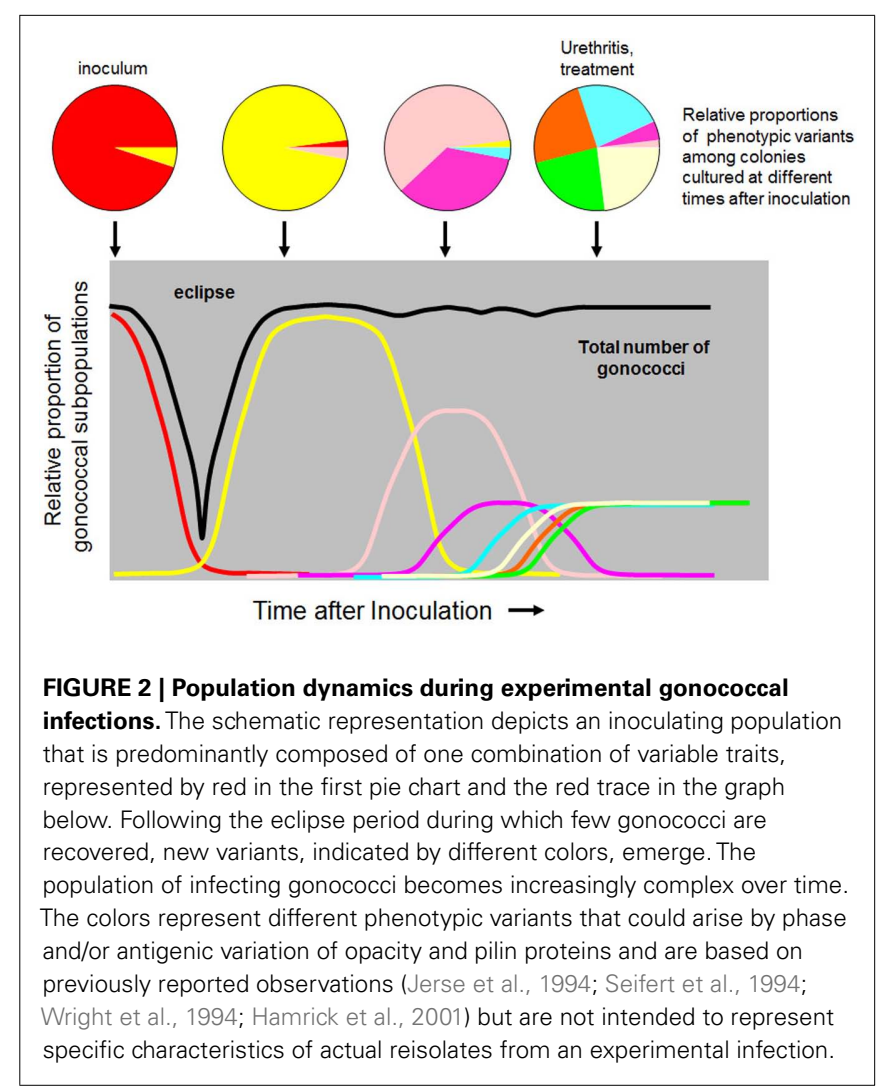

PilE and possibly other traits. The eclipse period that occurs immediately after inoculation could result in a population bottleneck, with the subsequent gonococcal population expanding from one or a few organisms present in the inoculum. New variants generated through phase and antigenic variation may descend from a single organism; these new variants proliferate if they express advantageous combinations of traits and eventually replace the previous population.

The selective pressures that could result in the sequential outgrowth and disappearance of gonococcal variants are not known. However, the possibility that very few bacteria emerge from a

Table 4 | Requirements for IgA protease and iron acquisition from human iron-binding proteins during experimental gonorrhea.

\begin{tabular}{|c|c|c|c|}
\hline Phenotype tested & Strain background & Experimental outcome & References \\
\hline Requirement for IgA protease activity & FA1090 & $\begin{array}{l}\text { Mutant unable to express IgA protease } \\
\text { as infectious as wild-type }\end{array}$ & Johannsen et al. (1999) \\
\hline $\begin{array}{l}\text { Requirement for lactoferrin (Lf) } \\
\text { utilization in the absence of transferrin } \\
\text { (Tf) utilization }\end{array}$ & FA1090 & $\begin{array}{l}\text { Mutant unable to use either Lf or Tf is } \\
\text { non-infectious }\end{array}$ & Cornelissen et al. (1998) \\
\hline $\begin{array}{l}\text { Ability to use Lf in the absence of Tf } \\
\text { utilization }\end{array}$ & FA1090 & $\begin{array}{l}\text { Mutant able to use Lf only as infectious } \\
\text { as wild-type that uses Tf only }\end{array}$ & Anderson et al. (2003) \\
\hline Ability to use both Lf and Tf & FA1090 & $\begin{array}{l}\text { Mutant able to use both Lf and Tf } \\
\text { showed competitive advantage over } \\
\text { wild-type in mixed infections }\end{array}$ & Anderson et al. (2003) \\
\hline
\end{tabular}

$\lg A$, immunoglobulin A; Lf, lactoferrin; Tf, transferrin. 
selective bottleneck to initiate infection following the initial eclipse period could confound interpretation of results from mixed experimental infections with isogenic strains differing in expression of a postulated virulence factor. Figure 3A shows theoretical outcomes of mixed infection initiated under conditions with and without the hypothetical bottleneck. In the absence of a dramatic population restriction, gonococcal populations reisolated from infected subjects would be expected to contain mixtures of the two strains in similar proportions to their representation in the inoculum, if they have equal fitness during infection (Pattern A). If one strain has a competitive advantage, this strain would eventually predominate among reisolated bacteria (Pattern B). If the bottleneck does reduce the infecting gonococcal population to very few bacteria, and one strain has a competitive advantage, that strain would be expected to predominate early among reisolates and be recovered exclusively from all infected subjects (Pattern C). If both input strains have equivalent fitness, each would be equally likely to survive the bottleneck, and each should predominate among reisolates from roughly half of infected subjects (Pattern D).

Figure 3B shows the actual results of mixed infections with wild-type Tf $+\mathrm{Lf}-$ and mutant $\mathrm{Tf}+\mathrm{Lf}+$ N. gonorrhoeae FA1090 strains. Perhaps not surprisingly, the data do not fit clearly into one single predicted outcome pattern. From over half of the infected subjects (3/5), only $\mathrm{Tf}+\mathrm{Lf}+$ gonococci were recovered following a noticeable eclipse. From the other two subjects, $\mathrm{Tf}+\mathrm{Lf}+$ gonococci eventually overtook the population after mixtures of both inoculating strains were recovered. The persistence of substantial numbers of both input strains with gradual out competition by the $\mathrm{Tf}+\mathrm{Lf}+$ strain in some subjects suggests that there is not a universal bottleneck limiting the establishment of experimental urethral infection to one or a few gonococci in the infecting population. The fact that cultures from all infected subjects ultimately contained $100 \% \mathrm{Tf}+\mathrm{Lf}+$ gonococci suggests that this strain had a selective advantage over the $\mathrm{Tf}+\mathrm{Lf}$ - wild-type strain during experimental infection of the male urethra. However, the population dynamics appeared to be different in different subjects. It is possible that there are individual differences in the effectiveness of innate host defenses, and these could affect the number of organisms in the original inoculum that survive and replicate to generate the infecting population.

Interpretation of mixed experimental infections could be further confounded by the potential for in vivo transformation between co-infecting gonococcal cells. In vitro models suggest that transformation during infection is possible, and the formation of mosaic genes is indirect evidence that it occurs in the human host. Gonococcal isolates that have been recovered to date from subjects inoculated with mixtures of isogenic strains showed no evidence of transformation during the relatively short experimental infection period (Anderson et al., 2003). However, only genetic exchanges that resulted in mosaic structures involving the engineered gene would have been detected. Recent improvements in whole genome sequencing technologies may enable a more comprehensive and sensitive approach to explore whether horizontal genetic exchange occurs during experimental urethral infection.

\section{SAFETY AND UTILITY OF THE EXPERIMENTAL HUMAN GONORRHEA MODEL}

Experimental infection of male volunteers with $N$. gonorrhoeae is safe, and subjects infected with wild-type gonococci exhibit the signs and symptoms of natural infection. The risks of serious complications from urethral gonococcal infection are extremely rare, even in natural infection. Hundreds of subjects have participated in experimental infection trials without severe adverse events. Using a competitive infection design with mixed inocula, the model can discriminate among gonococcal strains with different degrees of fitness for urethral colonization and infection. Experience with the FA1090 transferrin-receptor mutant showed that it is possible to render N. gonorrhoeae non-infectious by inactivation of a single virulence factor (in a strain that does not express the lactoferrin receptor). Thus the urethral infection model

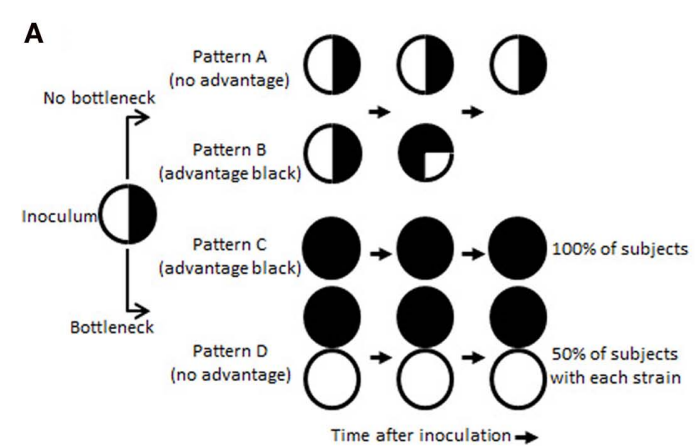

FIGURE 3 | Potential influence of population dynamics on outcomes of mixed experimental infections. In (A), theoretical outcomes are shown for infections initiated by a 50:50 mixture of two isogenic strains differing in expression of a predicted virulence factor, depicted as black and white, under conditions with (bottom) and without (top) a population-restricting bottleneck early after inoculation. Potential outcomes consistent with and without a competitive advantage for the black strain are indicated as four patterns of pie charts representing
B

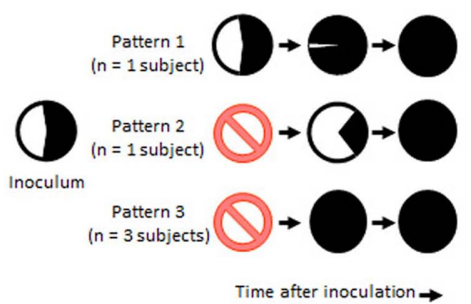

populations of gonococci recovered from infected subjects. In (B), actual outcomes are shown for infections initiated by a 45:55 mixture of isogenic $\mathrm{Tf}+\mathrm{Lf}-$ (white) and $\mathrm{Tf}+\mathrm{Lf}+$ (black) strains of $N$. gonorrhoeae FA1090 (Anderson et al., 2003). In patterns 1-3, each pie chart represents the mixture of gonococcal strains recovered from infected subjects over the 1- to 5-day period after inoculation. The $\oslash$ symbol indicates no gonococci were recovered at early times (1-3 days in different volunteers) after inoculation of four subjects 
is well-suited to provide insights into the mechanisms of pathogenesis of $N$. gonorrhoeae and eventually may prove useful as a component in the clinical development plan for the evaluation of a gonococcal vaccine.

\section{ACKNOWLEDGMENTS}

We are grateful to Janne G. Cannon for sharing previously unpublished data and for critically reviewing the manuscript. We thank

\section{REFERENCES}

Anderson, J. E., Hobbs, M. M., Biswas, G. D., and Sparling, P. F. (2003). Opposing selective forces for expression of the gonococcal lactoferrin receptor. Mol. Microbiol. 48, 1325-1337.

Anderson, J. E., Leone, P. A., Miller, W. C., Chen, C., Hobbs, M. M., and Sparling, P. F. (2001). Selection for expression of the gonococcal hemoglobin receptor during menses. $J$. Infect. Dis. 184, 1621-1623.

Apicella, M. A., Mandrell, R. E., Shero, M., Wilson, M. E., Griffiss, J. M., Brooks, G. F., Lammel, C., Breen, J. F., and Rice, P. A. (1990). Modification by sialic acid of Neisseria gonorrhoeae lipooligosaccharide epitope expression in human urethral exudates: an immunoelectron microscopic analysis. J. Infect. Dis. 162, 506-512.

Ayala, P., Vasquez, B., Wetzler, L., and So, M. (2002). Neisseria gonorrhoeae porin P1.B induces endosome exocytosis and a redistribution of Lamp1 to the plasma membrane. Infect. Immun. 70, 5965-5971.

Balthazar, J. T., Gusa, A., Martin, L. E., Chouhury, B., Carlson, R., and Shafer, W. M. (2011). Lipooligosaccharide structure is an important determinant in the resistance of Neisseria gonorrhoeae to antimicrobial agents of innate host defense. Front. Microbiol. 2:30. doi: 10.3389/fmicb.2011.00030

Banerjee, A., Wang, R., Uljon, S. N., Rice, P. A., Gotschlich, E. C., and Stein, D. C. (1998). Identification of the gene $(\lg t G)$ encoding the lipooligosaccharide beta chain synthesizing glucosyl transferase from Neisseria gonorrhoeae. Proc. Natl. Acad. Sci. U.S.A. 95, 10872-10877.

Bhat, K. S., Gibbs, C. P., Barrera, O., Morrison, S. G., Jahnig, F., Stern, A., Kupsch, E. M., Meyer, T. F., and Swanson, J. (1991). The opacity proteins of Neisseria gonorrhoeae strain MS11 are encoded by a family of 11 complete genes. Mol. Microbiol. 5, 1889-1901.
Cannon, J. G., Cohen, M. S., Isbey, S. F., Snodgrass, T. L., Wallace, A., Dempsey, J. A. F., Apicella, M., and Zhou, D. (1996). "Infectivity of gonococcal mutants in the human challenge model," in 10th International Pathogenic Neisseria Conference, Baltimore, MD.

Cohen, M. S., and Cannon, J. G. (1999). Human experimentation with Neisseria gonorrhoeae: progress and goals. J. Infect. Dis.179, S375-S379. E., Charniga, L. M., Isbey, S. F., and Whicker, L. G. (1994). Human experimentation with Neisseria gonorrhoeae: rationale, methods, and implications for the biology of infection and vaccine development. J. Infect. Dis. 169, 532-537.

Cole, J. G., Fulcher, N. B., and Jerse, A. E. (2010). Opacity proteins increase Neisseria gonorrhoeae fitness in the female genital tract due to a factor under ovarian control. Infect. Immun. 78, 1629-1641.

Connell, T. D., Shaffer, D., and Cannon, J. G. (1990). Characterization of the repertoire of hypervariable regions in the Protein II (opa) gene family of Neisseria gonorrhoeae. Mol. Microbiol. 4, 439-449.

Cornelissen, C. N., Kelley, M., Hobbs, M. M., Anderson, J. E., Cannon, J. G., Cohen, M. S., and Sparling, P. F. (1998). The transferrin receptor expressed by gonococcal strain FA1090 is required for the experimental infection of human male volunteers. Mol. Microbiol. 27, 611-616.

Dillard, J. P., and Seifert, H. S. (2001). A variable genetic island specific for providing DNA for natural transformation and is found more often in disseminated infection isolates. Mol. Microbiol. 41, 263-277.

Edwards, J. L., and Apicella, M. A. (2004). The molecular mechanisms used by Neisseria gonorrhoeae to initiate infection differ between men and women. Clin. Microbiol. Rev. 17, 965-981.

Edwards, M., McDade, R. L., Schoolnik, G., Rothbard, J. B., and Gotschlich,
Cohen, M. S., Cannon, J. G., Jerse, A. Neisseria gonorrhoeae is involved in

Christopher E. Thomas for sharing data in advance of publication. Experimental gonococcal infection studies at the University of North Carolina at Chapel Hill were supported by the Sexually Transmitted Infections Cooperative Research Center of the US National Institutes of Health (U19-AI031496). William M. Shafer was supported in part by a Senior Research Career Scientist Award from the Medical Research Service of the Department of Veterans Affairs.

E. C. (1984). Antigenic analysis of gonococcal pili using monoclonal antibodies. J. Exp. Med. 160, 1782-1791.

Elkins, C., Carbonetti, N. H., Varela, V. A., Stirewalt, D., Klapper, D. G. and Sparling, P. F. (1992). Antibodies to $\mathrm{N}$-terminal peptides of gonococcal porin are bactericidal when gonococcal lipopolysaccharide is not sialylated. Mol. Microbiol. 6, 2617-2628.

Elkins, C., and Rest, R. F. (1990). Monoclonal antibodies to outer membrane protein PII block interactions of Neisseria gonorrhoeae with human neutrophils. Infect. Immun. 58, 1078-1084.

Fischer, S. H., and Rest, R. F. (1988). Gonococci possessing only certain P. II outer membrane proteins interact with human neutrophils. Infect. Immun. 56, 1574-1579.

Fox, K. K., Thomas, J. C., Weiner, D. H., Davis, R. H., Sparling, P. F., and Cohen, M. S. (1998). Longitudinal evaluation of serovarspecific immunity to Neisseria gonorrhoeae. Am. J. Epidemiol. 149, 353-358.

Frieden, T. R., and Collins, F. S. (2010). Intentional infection of vulnerable populations in 1946-1948: another tragic history lesson. JAMA 304, 2063-2064.

Gill, M. J., McQuillen, D. P., van Putten, J. P., Wetzler, L. M., Bramley, J., Crooke, H., Parsons, N. J., Cole, J. A., and Smith, H. (1996). Functional characterization of a sialyltransferase-deficient mutant of Neisseria gonorrhoeae. Infect. Immun. 64, 3374-3378.

Griffiss, J. M., Jarvis, G. A., O’Brien, J. P., Eads, M. M., and Schneider, H. (1991). Lysis of Neisseria gonorrhoeae initiated by binding of normal human IgM to a hexosamine-containing lipooligosaccharide epitope(s) is augmented by strain-specific, properdinbinding-dependent alternative complement pathway activation. J. Immunol. 147, 298-305.
Hamrick, T. S., Dempsey, J. A., Cohen, M. S., and Cannon, J. G. (2001). Antigenic variation of gonococcal pilin expression in vivo: analysis of the strain FA1090 pilin repertoire and identification of the pilS gene copies recombining with pile during experimental human infection. Microbiology 147, 839-849.

Harvey, H. A., Jennings, M. P., Campbell, C. A., Williams, R., and Apicella, M. A. (2001). Receptor-mediated endocytosis of Neisseria gonorrhoeae into primary human urethral epithelial cells: the role of the asialoglycoprotein receptor. Mol. Microbiol. 42, 659-672.

Hosmer, D. W., and Lemeshow, S. (2000). Applied Logistic Regression. Hoboken, NJ: John Wiley \& Sons, Inc.

Hunter, J. (1835). "A treatise on venereal disease," in The Works of John Hunter, Vol. 2, ed. J. F. Palmer (London: Longman), 417.

James, J. F., and Swanson, J. (1978) Studies on gonococcus infection. XIII. Occurrence of color/opacity colonial variants in clinical cultures. Infect. Immun. 19, 332-340.

Jerse, A. E. (1999). Experimental gonococcal genital tract infection and opacity protein expression in estradiol-treated mice. Infect. Immun. 67, 5699-5708.

Jerse, A. E., Cohen, M. S., Drown, P. M., Whicker, L. G., Isbey, S. F., Seifert, H. S., and Cannon, J. G. (1994). Multiple gonococcal opacity proteins are expressed during experimental urethral infection in the male. J. Exp. Med. 179, 911-920.

Johannsen, D. B., Johnston, D. M., Koymen, H. O., Cohen, M. S., and Cannon, J. G. (1999). A Neisseria gonorrhoeae immunoglobulin Al protease mutant is infectious in the human challenge model of urethral infection. Infect. Immun. 67, 3009-3013.

John, C. M., Schneider, H., and Griffiss, J. M. (1999). Neisseria gonorrhoeae that infect men have lipooligosaccharides with terminal $\mathrm{N}$-acetyllactosamine repeats. J. Biol. Chem. 274, 1017-1025. 
Kellogg, D. S. Jr., Cohen, I. R., Norins, L. C., Schroeter, A. L., and Reising, G. (1968). Neisseria gonorrhoeae II. Colonial variation and pathogenicity during 35 months in vitro. J. Bacteriol. 96, 596-605.

Kellogg, D. S. Jr., Peacock, W. L. Jr., Deacon, W. E., Brown, L., and Pirkle, C. I. (1963). Neisseria gonorrhoeae. I. Virulence genetically linked to clonal variation. J. Bacteriol. 85, 1274-1279.

Kilian, M., Reinholdt, J., Lomholt, H., Poulsen, K., and Frandsen, E. V. (1996). Biological significance of IgA1 proteases in bacterial colonization and pathogenesis: critical evaluation of experimental evidence. APMIS 104, 321-338.

Kim, J. J., Zhou, D., Mandrell, R. E., and Griffiss, J. M. (1992). Effect of exogenous sialylation of the lipooligosaccharide of Neisseria gonorrhoeae on opsonophagocytosis. Infect. Immun. 60, 4439-4442.

Kirchner, M., and Meyer, T. F. (2005). The PilC adhesin of the Neisseria type IV pilus-binding specificities and new insights into the nature of the host cell receptor. Mol. Microbiol. 56, 945-957.

Lewis, D. A. (2010). The gonococcus fights back: is this time a knock out? Sex. Transm. Infect. 86, 415-421.

Lewis, L. A., Choudhury, B., Balthazar, J. T., Martin, L. E., Ram, S., Rice, P. A., Stephens, D. S., Carlson, R., and Shafer, W. M. (2009). Phosphoethanolamine substitution of lipid $\mathrm{A}$ and resistance of Neisseria gonorrhoeae to cationic antimicrobial peptides and complementmediated killing by normal human serum. Infect. Immun. 77, 1112-1120.

Lin, L., Ayala, P., Larson, J., Mulks, M., Fukuda, M., Carlsson, S. R., Enns, C., and So, M. (1997). The Neisseria type 2 IgAl protease cleaves LAMP1 and promotes survival of bacteria within epithelial cells. Mol. Microbiol. 24, 1083-1094.

Mandrell, R. E., Griffiss, J. M., and Macher, B. A. (1988). Lipooligosaccharides (LOS) of Neisseria gonorrhoeae and Neisseria meningitidis have components that are immunochemically similar to precursors of human blood group antigens. Carbohydrate sequence specificity of the mouse monoclonal antibodies that recognize crossreacting antigens on LOS and human erythrocytes. J. Exp. Med. 168, 107-126.
Mandrell, R. E., Lesse, A. J., Sugai, J. V., Shero, M., Griffiss, J. M., Cole, J. A., Parsons, N. J., Smith, H., Morse, S. A., and Apicella, M. A. (1990). In vitro and in vivo modification of Neisseria gonorrhoeae lipooligosaccharide epitope structure by sialylation. J. Exp. Med. 171, 1649-1664.

Mickelsen, P. A., Blackman, E., and Sparling, P. F. (1982). Ability of Neisseria gonorrhoeae, Neisseria meningitidis, and commensal Neisseria species to obtain iron from lactoferrin. Infect. Immun. 35, 915-920.

Nachamkin, I., Cannon, J. G., and Mittler, R. S. (1981). Monoclonal antibodies against Neisseria gonorrhoeae: production of antibodies directed against a strain-specific cell surface antigen. Infect. Immun. 32, 641-648.

Nassif, X., Beretti, J. L., Lowy, J., Stenberg, P., O'Gaora, P., Pfeifer, J., Normark, S., and So, M. (1994). Roles of pilin and PilC in adhesion of Neisseria meningitidis to human epithelial and endothelial cells. Proc. Natl. Acad. Sci. U.S.A. 91, 3769-3773.

Parsons, N. J., Andrade, J. R., Patel, P. V., Cole, J. A., and Smith, H. (1989). Sialylation of lipopolysaccharide and loss of absorption of bactericidal antibody during conversion of gonococci to serum resistance by cytidine 5'-monophosphoN-acetyl neuraminic acid. Microb. Pathog. 7, 63-72.

Parsons, N. J., Constantinidou, C., Cole, J. A., and Smith, H. (1994). Sialylation of lipopolysaccharide by CMP-NANA in viable gonococci is enhanced by low $\mathrm{Mr}$ material released from blood cell extracts but not by some UDP sugars. Microb. Pathog. 16, 413-421.

Rahman, M., Kallstrom, H., Normark, S., and Jonsson, A. B. (1997). PilC of pathogenic Neisseria is associated with the bacterial cell surface. Mol. Microbiol. 25, 11-25.

Ram, S., Cullinane, M., Blom, A. M., Gulati, S., McQuillen, D. P., Boden, R., Monks, B. G., O'Connell, C., Elkins, C., Pangburn, M. K., Dahlback, B., and Rice, P. A. (2001). C4bp binding to porin mediates stable serum resistance of Neisseria gonorrhoeae. Int. Immunopharmacol. 1, 423-432.

Ramsey, K. H., Schneider, H., Cross, A. S., Boslego, J. W., Hoover, D. L., Staley, T. L., Kuschner, R. A., and Deal, C. D. (1995). Inflammatory cytokines produced in response to experimental human gonorrhea. J. Infect. Dis. 172 , 186-191.

Rest, R. F., and Frangipane, J. V. (1992). Growth of Neisseria gonorrhoeae in CMP-N-acetylneuraminic acid inhibits nonopsonic (opacityassociated outer membrane protein-mediated) interactions with human neutrophils. Infect. Immun. 60, 989-997.

Rouquette, C., Harmon, J. B., and Shafer, W. M. (1999). Induction of the mtrCDE-encoded efflux pump system of Neisseria gonorrhoeae requires $\mathrm{MtrA}$, an AraClike protein. Mol. Microbiol. 33, 651-658.

Rudel, T., Scheurerpflug, I., and Meyer, T. F. (1995). Neisseria PilC protein identified as type-4 pilus tip-located adhesin. Nature 373, 357-359.

Rudel, T., van Putten, J. P., Gibbs, C. P., Haas, R., and Meyer, T. F. (1992). Interaction of two variable proteins (PilE and PilC) required for pilus-mediated adherence of Neisseria gonorrhoeae to human epithelial cells. Mol. Microbiol. 6, 3439-3450.

Sadarangani, M., Pollard, A. J., and Gray-Owen, S. D. (2011). Opa proteins and CEACAMs: pathways of immune engagement for pathogenic Neisseria. FEMS Microbiol. Rev. 35, 498-514.

Schmidt, K. A., Deal, C. D., Kwan, M., Thattassery, B. S., and Schneider, H. (2000). Neisseria gonorrhoeae MS11mkC opacity protein expression in vitro and during human volunteer infectivity studies. Sex. Transm. Dis. 27, 278-283.

Schmidt, K. A., Schneider, H., Lindstrom, J. A., Boslego, J. W., Warren, R. A., Van de Verg, L., Deal, C. D., McClain, J. B., and Griffiss, J. M. (2001). Experimental gonococcal urethritis and reinfection with homologous gonococci in male volunteers. Sex. Transm. Dis. 28, 555-564.

Schneider, H., Cross, A. S., Kuschner, R. A., Taylor, D. N., Sadoff, J. C., Boslego, J. W., and Deal, C. D. (1995). Experimental human gonococcal urethritis: 250 Neisseria gonorrhoeae MS11mkC are infective. J. Infect. Dis. 172, 180-185.

Schneider, H., Griffiss, J. M., Boslego, J. W., Hitchcock, P. J., Zahos, K. M., and Apicella, M. A. (1991). Expression of paragloboside-like lipooligosaccharides may be a necessary component of gonococcal pathogenesis in men. J. Exp. Med. 174, 1601-1605.

Schneider, H., Schmidt, K. A., Skillman, D. R., Van de Verg, L., Warren, R. L., Wylie, H. J., Sadoff, J. C., Deal, C. D., and Cross, A. S. (1996). Sialylation lessens the infectivity of Neisseria gonorrhoeae MS11mkC. J. Infect. Dis. 173, 1422-1427.

Seifert, H. S., Wright, C. J., Jerse, A. E., Cohen, M. S., and Cannon, J. G. (1994). Multiple gonococcal pilin antigenic variants are produced during experimental human infections. J. Clin. Invest. 93, 2744-2749.

Sheu, J. N., Chen, M. C., Cheng, S. L., Lee, I. C., Chen, S. M., and Tsay, G. J. (2007). Urine interleukin-1beta in children with acute pyelonephritis and renal scarring. Nephrology (Carlton) 12, 487-493.

Sheu, J. N., Chen, M. C., Lue, K. H., Cheng, S. L., Lee, I. C., Chen, S. M., and Tsay, G. J. (2006). Serum and urine levels of interleukin-6 and interleukin- 8 in children with acute pyelonephritis. Cytokine 36, 276-282.

Simms, A. N., and Jerse, A. E. (2005). "Role of phase and antigenic variation in Neisseria gonorrhoeae colonization," in Colonization of Mucosal Surfaces, eds J. P. Nataro, H. L. T. Mobley, and J. N. Weiser (Washington, DC: ASM Press), 326-250.

Swanson, J., Bergstrom, S., Barrera, O., Robbins, K., and Corwin, D. (1985). Pilus- gonococcal variants. Evidence for multiple forms of piliation control. J. Exp. Med. 162, 729-744.

Swanson, J., Robbins, K., Barrera, O., Corwin, D., Boslego, J., Ciak, J., Blake, M., and Koomey, J. M. (1987). Gonococcal pilin variants in experimental gonorrhea. J. Exp. Med. 165, 1344-1357.

van Vliet, S. J., Steeghs, L., Bruijns, S. C., Vaezirad, M. M., Snijders Blok, C., Arenas Busto, J. A., Deken, M., van Putten, J. P., and van Kooyk, Y. (2009). Variation of Neisseria gonorrhoeae lipooligosaccharide directs dendritic cell-induced $\mathrm{T}$ helper responses. PLoS Pathog. 5, e1000625. doi: 10.1371/journal.ppat.1000625

Virji, M. (2009). Pathogenic neisseriae: surface modulation, pathogenesis and infection control. Nat. Rev. Microbiol. 7, 274-286.

Warner, D. M., Shafer, W. M., and Jerse, A. E. (2008). Clinically relevant mutations that cause derepression of the Neisseria gonorrhoeae MtrC-MtrD-MtrE efflux pump system confer different levels of antimicrobial resistance and in vivo fitness. Mol. Microbiol. 70, 462-478. 
Wetzler, L. M., Barry, K., Blake, M. S., and Gotschlich, E. C. (1992). Gonococcal lipooligosaccharide sialylation prevents complement-dependent killing by immune sera. Infect. Immun. 60, 39-43.

Wright, C., Jerse, A. E., Cohen, M. S., Cannon, J. G., and Seifert, H. S. (1994). Nonrepresentative PCR amplification of variable gene sequences in clinical specimens containing dilute, complex mixtures of microorganisms. J. Clin. Microbiol. 32, 464-468.

$\mathrm{Wu}$, H., and Jerse, A. E. (2006). Alpha-2,3-sialyltransferase enhances Neisseria gonorrhoeae survival during experimental murine genital tract infection. Infect. Immun. 74, 4094-4103.

Conflict of Interest Statement: The authors declare that the research was conducted in the absence of any commercial or financial relationships that could be construed as a potential conflict of interest.

Received: 01 March 2011; paper pending published: 25 March 2011; accepted: 17 May 2011; published online: 31 May 2011.

Citation: Hobbs MM, Sparling PF, Cohen MS, Shafer WM, Deal CD and Jerse AE (2011) Experimental gonococcal infection in male volunteers: cumulative experience with Neisseria gonorrhoeae strains FA1090 and MS11mkC. Front. Microbio.
2:123. doi: 10.3389/fmicb.2011.00123 This article was submitted to Frontiers in Cellular and Infection Microbiology, a specialty of Frontiers in Microbiology. Copyright (c) 2011 Hobbs, Sparling, Cohen, Shafer, Deal and Jerse. This is an open-access article subject to a nonexclusive license between the authors and Frontiers Media SA, which permits use, distribution and reproduction in other forums, provided the original authors and source are credited and other Frontiers conditions are complied with. 